\title{
Development of an experimental optoelectronic device to study the amplitude of mandibular movements
}

\section{Reinaldo Missaka ${ }^{(a)}$ Lena Katekawa Adachi(b) Regina Tamaki (c) \\ Rosemary Sadami Arai Shinkai(d) Tomie Nakakuki de Campos ${ }^{(e)}$ Oswaldo Horikawa ${ }^{(f)}$}

(a) PhD Student; (b)PhD; (c) PhD, Professor; (e) PhD, Associate Professor - Department of Prosthodontics, School of Dentistry, University of São Paulo.

(d) PhD, Associate Professor, Department of Prosthodontics, Dental School, Pontifical Catholic University of Rio Grande do Sul.

(f) $\mathrm{PhD}$, Associate Professor, Department of Mechatronic Engineering, Polytechnic School, University of São Paulo.
Corresponding author:

Tomie Nakakuki de Campos

FOUSP - Depto. de Prótese Dentária

Av. Prof. Lineu Prestes, 2227

Cid. Universitária

São Paulo - SP - Brazil

CEP: 05508-000

E-mail:tncampos@usp.br

\begin{abstract}
This study aimed to present a wireless mandibular motion tracking device and optoelectronic data acquisition system developed to analyze the real-time spatial motion of the entire mandible during mouth opening and closing with no restriction of any movement. The procedures were divided into three phases: confection of a kinematic arch, dynamic digital video image acquisition, and image processing and analysis by using graphic computation. Four sequences of jaw opening/closing movements were recorded in lateral view: two from the maximum intercuspation (MIC) and the other two from a forced mandibular retruded position. Jaw motion was recorded by a digital video camera and processed as spatial coordinates corresponding to the position variation of the markers in the kinematic arch. The results showed that the method was capable of recording and processing the dynamics of the mandibular movements during jaw opening/closing using pixel-magnitude points. The mandible showed points with less displacement located near the temporomandibular joint during the opening/closing movements from the mandibular retruded position. When the jaw movements were recorded from MIC, these points were located near the mandibular foramen.
\end{abstract}

Descriptors: Movement; Temporomandibular joint; Mandible. 


\section{Introduction}

Analysis of the mandibular movements requires a comprehensive understanding of the mechanical and neuromuscular principles of the complex kinematics of the mandible and its related structures, i.e., muscles, ligaments, teeth and temporomandibular joints. Considerable advances have been achieved regarding dynamic biomechanical modeling, yet there still is a lack of a reliable and simple technique capable of recording and processing jaw movements near dental occlusion. ${ }^{1}$ For decades, mandibular movements have been assessed by using a number of different methods including graphic tracings, ${ }^{2,3}$ imaging (lateral radiographs), ${ }^{4-6}$ or electromagnetic transducers cemented on anterior teeth, ${ }^{7,8}$ but most devices often interfere in the complete closing of the mouth or in the maximum intercuspation (MIC) position, preventing the recording of jaw movements from the centric position.

Motion tracking systems based on optoelectronic technology become the preferred method to study jaw movements because of their operational and accuracy advantages over the other methods. ${ }^{9-15}$ For example, Karlsson et al. ${ }^{16}$ (1991) analyzed the velocity and the amplitude of jaw movements using light emitting diodes (LEDs) fixed on teeth in different positions that emitted controlled pulses of infrared light. The light was captured using a video camera and processed by means of a computer software as spatial coordinates with satisfactory precision. Koyano et al. ${ }^{17}$ (1997) also used a jaw-tracking device equipped with LEDs, but despite being fabricated with a light alloy of aluminum, the device had large dimensions affecting the jaw movement recording. Most of the reported optoelectronic systems for jaw motion recording were manufactured using LEDs or reflexive markers, which increased the cost and the complexity of image acquisition. Furthermore, some devices use cables to connect the jaw tracking device to the image acquisition unit, which may interfere with the mandibular movements.

This study aimed to present a wireless mandibular motion tracking device and optoelectronic data acquisition system capable of analyzing, by means of graphic computation, the real-time spatial behavior of the entire mandible during mouth opening and closing with no restriction of any movement.

\section{Material and Methods}

This study was approved by the institutional review board according to national and international standards (Declaration of Helsinki) for conducting studies with human subjects. One subject was selected according to the inclusion criteria: little or no alteration of the natural dental anatomy, Angle Class I occlusion, absence of any signs or symptoms of temporomandibular disorders.

The procedures were divided into three phases: confection of a kinematic arch, dynamic digital video image acquisition, and image processing and analysis using graphic computation.

\section{Kinematic arch}

The kinematic arch was designed to visually project to the extra-oral region the movements of the mandibular dental arch during jaw movement. The kinematic arch was composed of three parts: an intraoral metallic framework, an extra-oral arch, and markers for camera motion tracing (Figure 1).

Two metallic frameworks were fabricated in cobalt-chrome alloy, similarly to the fabrication procedure of removable partial prostheses (Figure 2), with four retention clasps (two posterior ones in the third molar region, and two anterior ones in the canine region), and four connectors for fixation of the extra-oral arch on the clasps. The position and fixation of the clasps and connectors were designed to prevent any interference with the centric positions or mandibular movements.

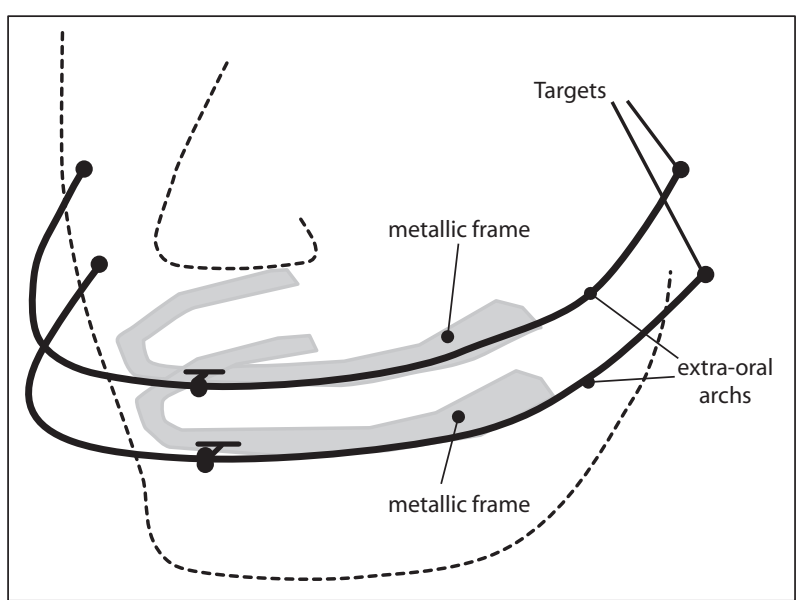

Figure 1 - Kinematic arch. 


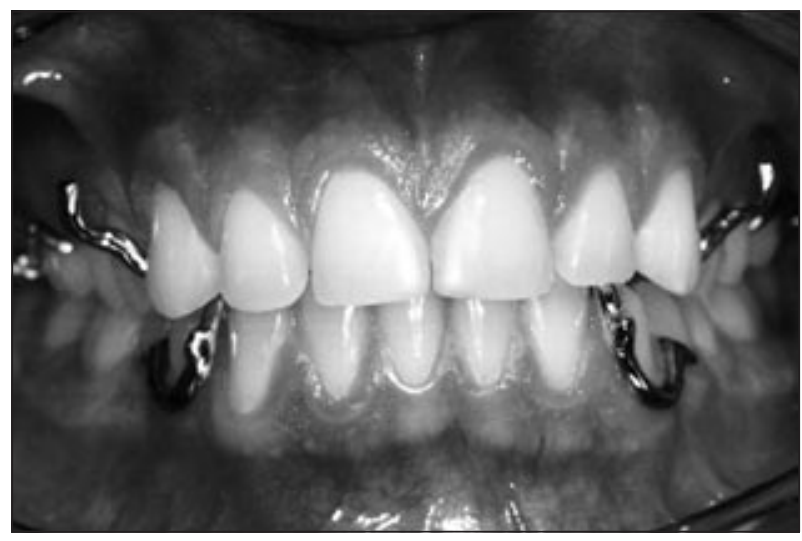

Figure 2 - Metallic frameworks with the retention clasps and the connectors in position.

An orthodontic extra-oral arch (standard 0.051"$1.30 \mathrm{~mm}$ ) was adapted and fixed to each of the metal frames on the connectors through laser welding. Small acrylic white spheres were used as landmarks (targets) and were fixed on opaque black suede paper used as a background to facilitate target recognition during tracing with the digital video camera. Two dark backgrounds with the targets were fixed to the lower extra-oral arch unilaterally in random positions, maintaining a maximum distance between each other, so as to remain equidistant in a parallel plane to the surface of the video camera lens. In this same manner, the other two targets were fixed on the superior extra-oral arch (Figure 3).

\section{Image acquisition and processing}

During the jaw opening/closing movements, the maxillary arch accompanied the cranium movement while the mandibular arch followed the jaw movement. The subject was instructed to open and close his mouth slowly while a series of images were obtained with the digital video camera. For each image frame recorded, the position of the targets was determined by image processing. Combining the movements recorded by the camera, the movement of the targets was acquired, and consequently a spatial representation of the mandibular movement was assembled.

After determining the geometric centers of the targets, resultant from the image processing, the trajectories and movement curves could be estab-

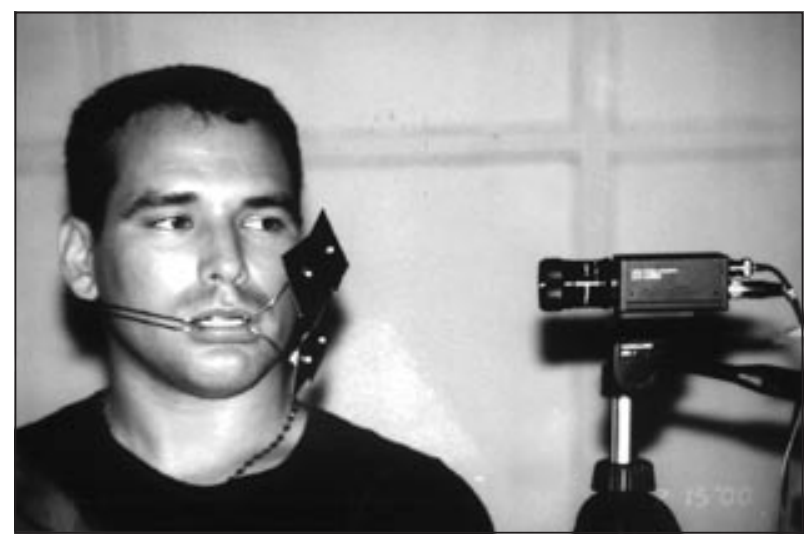

Figure 3 - Targets on the dark backgrounds fixed to the extra-oral arches.

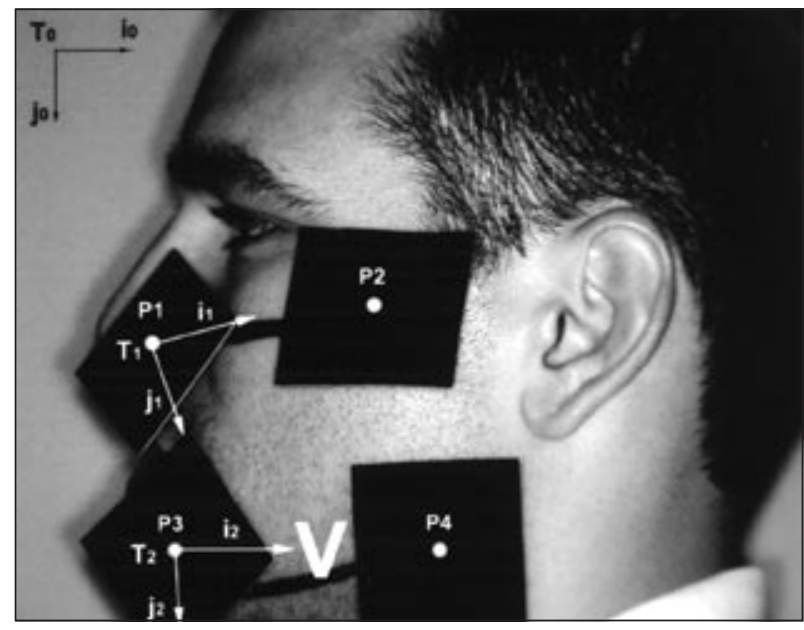

Figure 4 - System of coordinates T1 and T2.

lished. Original data were converted to the relative movement of the targets fixed on the mandible, with subtraction of any head movement, recorded by the pathways of the points fixed on the maxilla.

A file was obtained containing the coordinates of the center of gravity of each of the targets during filming. For each image identified by the period in time in which it was recorded $(t=0, t=1, t=2, \ldots$, $t=29$ ), the following elements were established (Figure 4):

- $\mathrm{P} 1(\mathrm{t}), \mathrm{P} 2(\mathrm{t}), \mathrm{P} 3(\mathrm{t}), \mathrm{P} 4(\mathrm{t})$ : points corresponding to the center of gravity of the targets;

- T0: system of global coordinates of the image, whose origin was the superior corner of the same; 
- T1: system of coordinates whose quaternion i1 was parallel to the P1P2 segment;

- T2: system of coordinates whose quaternion i2 was parallel to the P3P4 segment;

- V: regions whose points were supposedly accordant to the mandible, that is, all of the points in region $\mathrm{V}$ had constant coordinates in relation to T2.

For each image frame, the coordinates of the target center of gravity were determined and identified as:

$P 1-\left[x_{p 1}(t), y_{p 1}(t)\right]^{T 0} ; P 2-\left[x_{p 2}(t), y_{p 2}(t)\right]^{T 0} ; P 3-\left[x_{p 3}(t), y_{p 3}(t)\right]^{T 0} ; P 4-\left[x_{p 4}(t), y_{p 4}(t)\right]^{T 0}$

for $\mathrm{t}=0,1, \ldots, 29$, to indicate that these coordinates referred to the T0 system of coordinates. After processing a complete sequence of images (30 frames) for each video recording, a file was generated containing a sequence in time of the coordinates of the centers of gravity.

The data in this file was organized in the following manner:

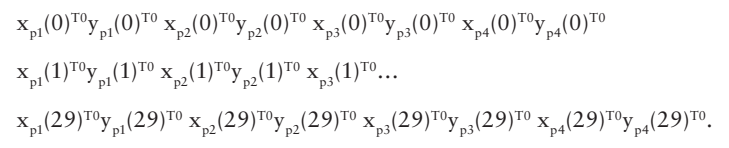

These files contained the points of the trajectories followed by P1 and P2 (of the T1 system of coordinates) and by P3 and P4 (of the T2 system of coordinates), during the time intervals, which varied between 0 and 29 .

The T1 system of coordinates represented the reference for movement analysis of the targets fixed on the mandible. Initially, region $\mathrm{V}$ was defined in the T2 system of coordinates; however, in order to obtain data that was not influenced by head movement, it was necessary to discover the movements of the $\mathrm{P}$ points that belonged to $\mathrm{V}$ in relation to the $\mathrm{T} 1$ system of coordinates.

For each of the image frames, the T1 (maxillary) and T2 (mandibular) systems of coordinates were in different positions in relation to the $\mathrm{T} 0$ system of coordinates due to the movements of the maxilla and mandible. Therefore, two coordinate transformations were needed for each image in order to obtain

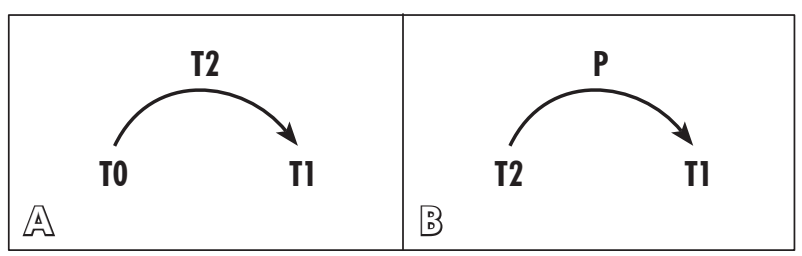

Figure $\mathbf{5}$ - A represents how the movement of the T2 system of coordinates was determined. B represents how the position of point $\mathrm{P}$ in $\mathrm{T} 1$ was determined.

the T2 points without the interference of T1 movement (Figure 5).

In this manner, a sequence of point $\mathrm{P}$ coordinates belonging to $\mathrm{V}$ were obtained, corresponding to the times of $\mathrm{T}=0,1, \ldots, 29$, expressed in the $\mathrm{T} 1$ system of coordinates.

A computer script was developed using the MATLAB environment for analysis of the point pathways. The points that expressed least variation in their position were identified aiming to determine the center of mandibular rotation. In addition, points with no dislocation in a given time interval were identified, which characterizes an instantaneous center of rotation.

The natural opening and closing of the mouth movement was performed from the MIC position (free movement), as well as from a forced retruded position of the mandible (forced movement), which was obtained with retrusive manipulation of the mentum region by the operator. The patient was oriented to execute the opening/closing jaw movement within an estimated time of 8 seconds to guarantee that the entire course of mandibular movement would be captured by the video camera.

Each coordinate file was submitted to analysis using the developed computer script, creating a new file which represented the points that suffered least spatial alteration during the movement of mouth opening and closing.

\section{Results}

In relation to the first image obtained at the beginning of the acquisition process using the video camera, graphs were mapped for the absolute target movements (light gray), the relative movements of the targets fixed to the mandible (dark gray) and 


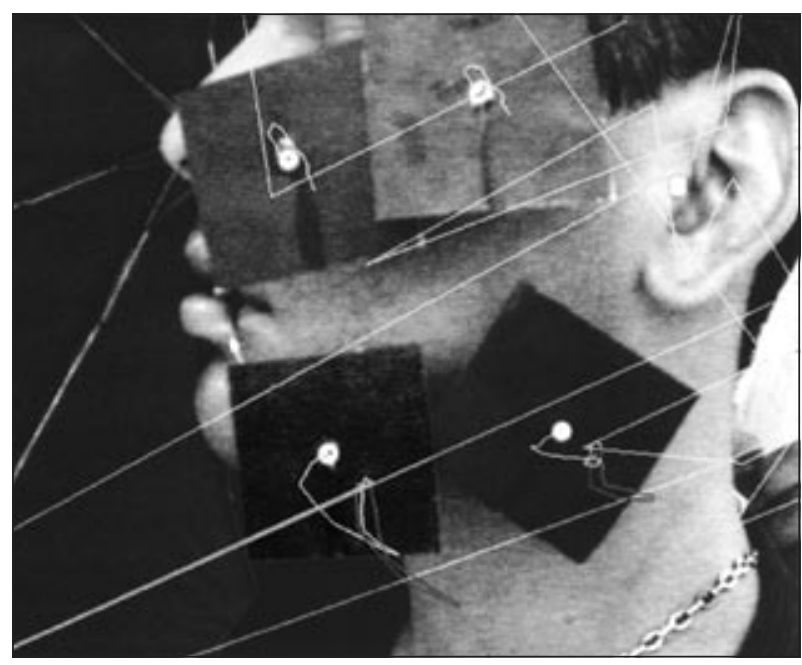

Figure 6 - Computer screen containing the final result of image 1 processing.

the points associated to the pixels that suffered less dislocation. The tables with the $(\mathrm{x}, \mathrm{y})$ values of pixel coordinates generated very large files, and, therefore, they are not presented.

\section{Acquisition of image 1 - forced movement}

Figure 6 shows the computer screen with the final result of image 1 processing, with the movement of opening and closing under mandibular retrusion. Note the points with the dimension of 1 pixel marked in the pre-auricular region close to the TMJ, which represent the points that had the least spatial variation (displacement) in the plane, yet all points had movement.

\section{Acquisition of image 2 - free movement}

Figure 7 shows the computer screen with the final result of image 2 processing, with the movement origin at the MIC position. Note the points with the dimensions of 1 pixel, marked in the mandibular angle, which represent the points that suffered least spatial variation (displacement) in the plane; however, all points also had movement.

\section{Discussion}

This study presented a prototype of a wireless, optoelectronic jaw motion technique and the development of a custom software to analyze mandibular movements in two-dimensional coordinates. The

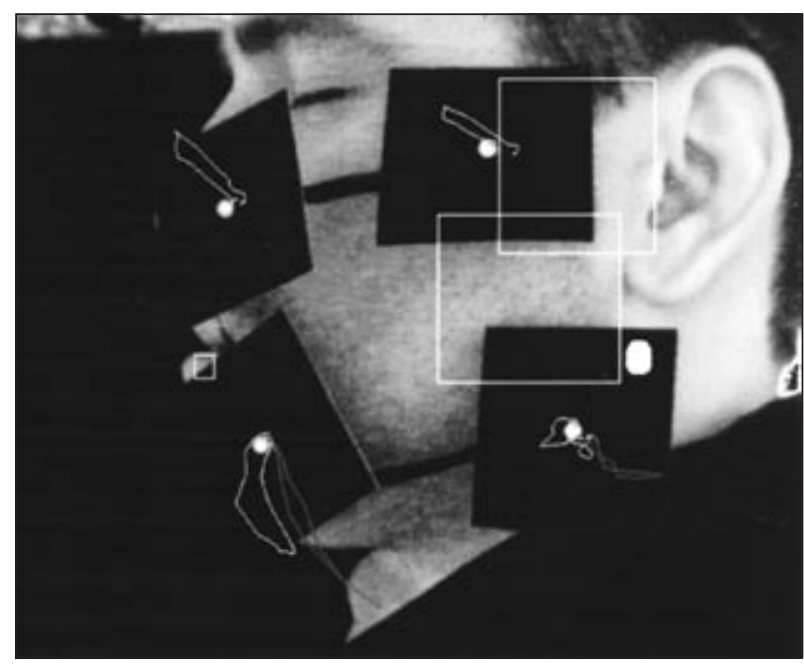

Figure 7 - Computer screen containing the final result of image 2 processing.

technique proved capable of recording point coordinates of 1-pixel magnitude. While analysing mandibular movements, high precision recording of the jaw pathways is mandatory. In some techniques that use LEDs as landmarks, ${ }^{16-18}$ these targets produce unreliable graphic tracings of mandibular movements because it is not possible to calculate the geometric center of the lights.

Another important requirement for the study of mandibular kinetics is the reproduction of natural movements, without difficulties or obstacles by the patient. The method developed in this study is simple to perform and does not require a physical connection between the targets and the mandible, or cables that may interfere with normal jaw movements. ${ }^{7,16,18}$ Several studies use landmarks fixed to incisors, $, 8,13,15,19$ which may affect the jaw motion pattern close to teeth contact.

The geometric centers, or centers of gravity obtained during mandible movement are associated to a system of absolute image coordinates, connected to the reference axes of the camera. They do not represent the real movement of the mandible. In order to obtain precise data, there should not be any head movement in relation to the camera. However, immobilizing the head to the support structure of the camera would not generate natural movements. In the present study, the head movement was subtracted from the original data because mandibular 
and head-neck movements are combined during jaw opening and closing. During mastication, the relative movement between the bodies invalidates the use of these axes as inertial reference positions. Because the mandible is connected to the cranium, the inertial reference to be adopted should be the cranium, and obtaining the coordinates of the shifting body (mandible) in relation to the fixed body (cranium) is enough for determining this measure. The coordinates of the mandibular target trajectories were transferred to the trajectory plane of the fixed maxillary targets using analytic equations of the studied points for acquisition of its relative position. This procedure rendered good results, and the final version of the program was able to extract the relative movements of the targets for the entire mandible. This allowed a more comprehensive analysis of the mandibular motion compared to that allowed by studies that focused on the jaw movement at the incisor point. ${ }^{19,20}$

During the movements of opening and closing with manual retrusion of the mandible, the 1-pixel points that suffered least spatial dislocation were those closest to the TMJ region. This localization could provide support for the debate about terminal axis rotation (Bisagra axis) as being situated in the condyles.

However, in order for this forced movement to produce a fixed central axis, or one that is located in the condyles, it would be necessary for at least one point to remain immobilized at some moment during image acquisition. The mathematical processing of the coordinates obtained from the movement of the targets, in the order of pixels, did not find any point that assumed this condition in any time interval.

The results obtained from the 2 images demonstrated that, from the MIC position, the 1-pixel points that had the least spatial dislocation were located near the mandibular angle region. This observation is in accordance with early findings that the mandibular rotation axis is near the mandibular foramen. ${ }^{21}$ Yet, using the present method, it is not possible to affirm the existence of a rotation axis near the mandibular foramen, which would require that at least one of the points remain immobile during a given moment of image acquisition, a condition that did not occur.

Coordinate analysis of all of the images demonstrated that it is possible to identify the kinematic behavior of all of the points on the patient's face on a computer screen in a defined plane. The evaluation method used in the present study for image processing and computer graphics, without the influence of possible errors from the operator, generates a good level of reliability and fidelity, in comparison to the photographic and radiographic methods reported in the literature. Furthermore, the order of magnitude of pixels used in the computerized processing allows greater precision.

This study has some limitations because it is a preliminary report of a new test system. The next step is to improve the image recording in a three-dimensional fashion and validate the system in normal and non-normal patients. These issues will be addressed in further studies.

\section{Conclusions}

In summary, we found that the developed method is capable of dynamically capturing and processing the movement of mandibular opening and closing in a two-dimensional plane, with a pixel-level accuracy. Furthermore, the method it uses for controlling errors and imprecision makes it reliable.

The mandible had points that suffered less dislocation, located next to the TMJ region, when the movements of mandibular opening and closing with retrusion were executed. The points with less dislocation during the natural movements of mouth opening and closing, from the MIC position, were located near the mandibular foramen. However, instantaneous centers of rotation could not be identified.

\section{Acknowledgements}

This study was partially supported by the Brazilian Ministry of Education/CAPES through a graduate scholarship. 


\section{References}

1. Koolstra JH. Dynamics of the human masticatory system. Crit Rev Oral Biol Med. 2002;13(4):366-76.

2. Gysi A. The problem of articulation. Dent Cosmos. 1910;52:119.

3. McCollum BB. Fundamentals involved in prescribing restorative dental remedies. Dent Items Interest. 1939;61:522-35.

4. Alexander PC. Movements of the condyle from rest position to initial contact and full occlusion. J Am Dent Assoc. 1952;45(3):284-93.

5. L'Estrange PR. A tomographic study of the hinge axis. Dent Pract Dent Rec. 1969;19:209-14.

6. Posselt U. Hinge opening axis of the mandible. Acta Odontol Scand. 1956;14:49-63.

7. Gibbs CH. Functional movements of the mandible. J Prosthet Dent. 1971;26:604-19.

8. Yoon HJ, Zhao KD, Rebellato J, An KN, Keller EE. Kinematic study of the mandible using an electromagnetic tracking device and custom dental appliance: introducing a new technique. J Biomech. 2006;39(12):2325-30.

9. Airoldi RL, Gallo LM, Palla S. Precision of the jaw tracking system JAWS 3D. J Orofac Pain. 1994;8(2):155-64.

10. Catic A, Naeije M. Location of the hinge axis and the kinematic centre in asymptomatic and clicking temporomandibular joints. J Oral Rehabil. 1999;26(8):661-5.

11. Ferrario VF, Sforza C, Lovecchio N, Mian F. Quantification of translational and gliding components in human temporomandibular joint during mouth opening. Arch Oral Biol. 2005;50(5):507-15.
12. Gallo LM, Airoldi GB, Airoldi RL, Palla S. Description of mandibular finite helical axis pathways in asymptomatic subjects. J Dent Res. 1997;76(2):704-13.

13. Jemt T. Masticatory mandibular movements. Analysis of a recording method and influence of the state of the occlusion. Swed Dent J. 1984;23(Suppl):1-52.

14. Maeda Y, Okada M, Mori T, Enomoto K, Sogo M, Okuno Y. Development of a mandibular tracking device with six degrees of freedom using optoelectronic system. J Osaka Univ Dent Sch. 1992;32:45-50.

15. Travers KH, Buschang PH, Hayasaki H, Throckmorton GS. Associations between incisor and mandibular condylar movements during maximum mouth opening in humans. Arch Oral Biol. 2000;45(4):267-75.

16. Karlsson S, Persson M, Carlsson GE. Mandibular movement and velocity in relation to state of dentition and age. J Oral Rehabil. 1991;18(1):1-8.

17. Koyano K, Ogawa T, Suetsugu T. The influence of canine guidance and condylar guidance on mandibular lateral movement. J Oral Rehabil. 1997;24(11):802-7.

18. Karlsson S, Carlsson GE. Recording of masticatory mandibular movements and velocity by an optoelectronic method. Int J Prosthodont. 1989;2(5):490-6.

19. Kang JH, Chung SC, Fricton JR. Normal movements of mandible at the mandibular incisor. J Prosthet Dent. 1991;66(5):687-92.

20. Jankelson B. Measurement accuracy of the mandibular kinesiograph - a computerized study. J Prosthet Dent. 1980;44(6):656-66.

21. Frank L. Opening axis of the jaw. Dent Dig. 1956;62:16-9. 